\title{
Magnetic proximity effect and superconducting triplet correlations at the cuprate superconductor and oxide spin valve interface
}

\author{
G. A. Ovsyannikov ${ }^{a)}$
}

Kotelkinov Institute of Radio Engineering and Electronics, RAS, Moscow 125009, Russia and Chalmers

University of Technology, Gothenburg SE-41296, Sweden

K. Y. Constantinian and V. V. Demidov

Kotelkinov Institute of Radio Engineering and Electronics, RAS, Moscow 125009, Russia

\section{Yu. N. Khaydukov}

Max-Planck Institute for Solid State Research, Stuttgart D-70569, Germany

(Submitted May 13, 2016)

Fiz. Nizk. Temp. 42, 1115-1128 (October 2016)

\begin{abstract}
A heterostructure consisting of a cuprate superconductor $\mathrm{YBa}_{2} \mathrm{Cu}_{3} \mathrm{O}_{7-\delta}$ and a ruthenate/manganite $\left(\mathrm{SrRuO}_{3} / \mathrm{La}_{0.7} \mathrm{Sr}_{0.3} \mathrm{MnO}_{3}\right)$ spin valve was studied using SQUID magnetometry, ferromagnetic resonance, and neutron reflectometry. It is shown that because of the magnetic proximity effect a magnetic moment is excited in the superconducting portion of the heterostructure, whereas the magnetic moment in the spin valve becomes suppressed. The experimentally obtained value of a typical penetration depth of a magnetic moment into the superconductor is significantly greater than the coherence length of the cuprate superconductor, which indicates that the induced magnetic moment mechanism of $\mathrm{Cu}$ atoms is dominant. The mesastructure prepared by adding niobium film as a second superconducting electrode to the existing heterostructure, exhibited a superconducting current (dc Josephson effect) at interlayer thicknesses that are much greater than the coherence length of the ferromagnetic materials. The maximum of the critical current density dependence on the thickness of the spin valve material corresponds to the interlayer coherence length, which agrees with the theoretical predictions associated with spin-triplet pairing. The superconducting current is observed at magnetic fields that are two orders of magnitude greater than the field corresponding to the occurrence of one magnetic flux quantum in the mesastructure. The ratio of the second harmonic of the current-phase dependence of the mesastructure superconducting current to the first, determined according to the dependence of the Shapiro steps on the amplitude of microwave exposure, did not exceed 50\%. Published by AIP Publishing. [http://dx.doi.org/10.1063/1.4966622]
\end{abstract}

\section{Introduction}

During the contact of a superconductor $(\mathrm{S})$ with a normal (non-superconducting) metal $(\mathrm{N})$ superconducting correlations penetrate to a distance that is much grater than the interatomic. ${ }^{1}$ This phenomenon has been dubbed the proximity effect, and was discussed in detail for the first time by de Gennes. ${ }^{2,3}$ Along with the penetration of superconducting correlations into the normal metal, there is a change to the superconducting order parameter in a superconductor due to "leakage of Cooper pairs." Earlier it was assumed that because of the "antagonism" between superconductivity and magnetism, there is no proximity effect at the superconductor (S) and ferromagnet (F) interface. This is due to the structure of the Cooper pairs, in which two electrons with opposing spins become coupled. Larkin and Ovchinnikov, ${ }^{4}$ as well as Fulde and Ferrell ${ }^{5}$ were the first to predict the occurrence of inhomogeneous superconducting correlations in ferromagnets (LOFF state). Even though the depth of superconducting correlation penetration into the ferromagnet is small, it was possible to experimentally prove the presence of the LOFF states in the S-F-S contact, manifesting themselves as oscillations of the superconducting critical current in the contact. ${ }^{6,7}$

In 2001 it was theoretically demonstrated that triplet superconducting correlations (TSC) occur together with the usual (singlet) correlations with non-zero spin projection, ${ }^{8,9}$ in a ferromagnet near an interface with a superconductor under the condition of spatial non-uniformity of ferromagnet magnetization. A distinctive feature of TSC is the fact that they are insensitive to the exchange field and penetrate deep into the ferromagnet at distances that are typical for a normal (nonmagnetic) metal, reaching hundreds of nm. Experimentally, the occurrence of TSC was recorded according to the presence of a superconducting current in structures composed of two superconductors with singlet superconductivity, coupled by a ferromagnetic interlayer with spiral magnetization, ${ }^{10}$ as well as a ferromagnetic film with non-uniform magnetization at the interface with the superconductor. ${ }^{11}$ TSCs in superconducting structures with a ferromagnetic interlayer made of two ferromagnets $(\mathrm{S} / \mathrm{FL} / \mathrm{FR} / \mathrm{S})$ were theoretically predicted with ballistic electron transport, ${ }^{12}$ and diffuse scattering. ${ }^{13}$ It was theoretically demonstrated that a dominant second harmonic in the current-phase dependence (CPD) of a superconducting transition current ${ }^{10,12-14}$ is one of the ways in which the longrange proximity effect manifests itself.

In oxide structures such as a cuprate superconductormanganite ferromagnet, the transparency of the interface is determined by the work function, ${ }^{15}$ and can be relatively low, which limits the proximity effect. Because of this, any 
reports about the excitation of triplet correlations at the cuprate superconductor and manganite ferromagnet interface are rather contradictory. ${ }^{16-21}$ It should be noted that the manganite ferromagnets $\mathrm{La}_{0.7} \mathrm{Sr}_{0.3} \mathrm{MnO}_{3}$ (LSMO) and $\mathrm{La}_{0.7} \mathrm{Ca}_{0.3} \mathrm{MnO}_{3}$ (LCMO) used in the experiment are ferromagnets having $100 \%$ carrier polarization (magnetic semimetal) at low temperatures, and therefore the appearance of singlet excitations at the ferromagnet boundary is suppressed, which does not exclude the excitation of spin-triplet correlations.

In the case of ferromagnet contact with a normal metal, the ferromagnetic correlations from the ferromagnet penetrate into the normal metal by a small (close to interatomic) distance due to the locality of the exchange interaction (see Refs. 22 and 23 for example). In Refs. 24-27 it was theoretically demonstrated that in a superconductor, at the boundary with a ferromagnet and under the influence of an exchange field, there is a change in the density of states due to its difference for electrons with spin-up and electrons with spindown. Later it was also theoretically demonstrated that the sign and magnitude of the magnetic moment occurring in the superconductor both strongly depend on the parameters of the $\mathrm{S} / \mathrm{F}$ border, such as boundary transparency, the presence of impurities, and layer thickness. ${ }^{28-31}$ An experimental study of the magnetic proximity effect in S/F structures based on metallic ferromagnets and conventional superconductors, performed using a variety of methods (ferromagnetic resonance, muon scattering, neutron scattering, etc. ${ }^{32-35}$ generally confirmed the conclusions of the theory.

Of the structures based on cuprate superconductors with a small coherence length and anisotropic superconducting gap, the most heavily studied are $\left[\mathrm{YBa}_{2} \mathrm{Cu}_{3} \mathrm{O}_{7} / \mathrm{La}_{2 / 3} \mathrm{Ca}_{1 /}\right.$ $\left.{ }_{3} \mathrm{MnO}_{3}\right]_{n}\left([\mathrm{YBCO} / \mathrm{LCMO}]_{n}\right)$ superlattices, which revealed the presence of a magnetic moment in the superconductor. $^{36-40}$ An induced magnetic moment of the $\mathrm{Cu}$ atoms, oriented in a direction that is antiparallel to the Mn atoms, was detected at the boundary between the cuprate superconductor and the magnetic material using X-ray dichroism. ${ }^{38-40}$ It was shown that the $\mathrm{Cu}$ and $\mathrm{Mn}$ atoms were connected through the interface by a covalent chemical bond, resulting in a strong hybridization and orbital reconstruction. Note that the typical lengths of the orbital reconstruction greatly exceed the interatomic distances and are equal to $8-10 \mathrm{~nm} .^{41,42}$

This paper is an experimental investigation of the changes to the magnetic moment of the heterostructure containing the cuprate superconductor-ferromagnetic spin valve (formed out of two ferromagnetic layers). Measurements of the heterostructure's magnetic moment were carried out with a SQUID magnetometer, neutron reflectometry and using ferromagnetic resonance (FMR). Analysis of the data obtained by way of these three methods allowed us to determine the magnitude of the magnetic moment induced in the superconductor, as well as the change to the magnetic moment of the ferromagnetic spin valve. The paper also presents the results of experimental studies of superconducting and quasiparticle currents in micron size mesastructures, prepared out of $\mathrm{Au} / \mathrm{LSMO} / \mathrm{SRO} /$ YBCO heterostructures with a top electrode made of $\mathrm{Nb}$. We evaluated characteristic values, such as the depth of the penetration of superconducting correlations into a ferromagnet and the transparency of the cuprate supeconductor-ruthenate ferromagnet border, and experimentally determined the contribution of the CPD second harmonic of the superconducting current. Based on the experimental data we concluded that the contribution of the triplet superconducting correlations to the transport of superconducting carriers in mesastructures is dominant.

\section{Experimental procedure and samples}

We studied epitaxial thin-film heterostructures consisting of a cuprate superconductor $\mathrm{YBa}_{2} \mathrm{Cu}_{3} \mathrm{O}_{7-\delta}$ (YBCO) and two ferromagnetic layers $\mathrm{SrRuO}_{3} \quad(\mathrm{SRO})$ and $\mathrm{La}_{0.7} \mathrm{Sr}_{0.3} \mathrm{MnO}_{3}$ (LSMO), prepared by laser ablation at temperatures of $700^{\circ}-800^{\circ} \mathrm{C}$ and oxygen pressure of $0.3-0.6$ mbar. The thickness of the superconductor is in the range of $80-200 \mathrm{~nm}$, whereas the thickness of the ferromagnetic layers varied from 5-20 nm (see Table 1). The heterostructures were covered by thin $(20 \mathrm{~nm})$ layer of gold on top. We used substrates with the dimensions $5 \times 5 \mathrm{~mm}$ made of $(110) \mathrm{NdGaO}_{3}(\mathrm{NGO})$, (001) $\mathrm{LaAlO}_{3}$ (LAO), and $\left(\mathrm{LaAlO}_{3}\right)_{0.3}+\left(\mathrm{Sr}_{2} \mathrm{AlTaO}_{6}\right)_{0.7}$ (LSAT). The magnetization vector of the LSMO epitaxial film deposited on a (110)NGO substrate or onto a (001)YBCO film, is generally in the plane of the substrate, ${ }^{43,44}$ whereas for the SRO film the magnetization vector is outside of the plane of the substrates used. ${ }^{45}$

A detailed study of the field and temperature dependences of magnetization of the separate films and heterostructures was conducted using a SQUID-magnetometer MPMS3 produced by Quantum Design in the VSM mode. ${ }^{46}$ The plane of the substrate was set relative to the direction of the magnetic field within $1^{\circ}-2^{\circ}$.

The heterostructures were also studied using a Bruker ER 200 magnetic resonance spectrometer, operating in the frequency $\omega / 2 \pi=9.7 \mathrm{GHz}$. We measured ferromagnetic resonance spectra over a wide range of temperatures: 20-300 K. The FMR spectra of the LSMO film in the heterostructures were obtained by cooling the sample in the field of the Earth. Upon reaching the given temperature we held a scanning of the magnetic field from 0 to $4 \mathrm{kOe}$. The FMR spectrum from the SRO film is invisible at our experimental conditions due to the large value of the magnetic anisotropy field of the SRO film.

The experiment with polarized neutrons was conducted on a NREX monochromatic reflectometer (wavelength $0.43 \mathrm{~nm}$, energy $4.4 \mathrm{meV}$ ), located at the research reactor FRM II (Garching, Germany). A beam of polarized neutrons

TABLE 1. The composition and thickness of the test heterostructures, as well as the experimentally determined values of the changes to the magnetic moment of the heterostructures. $d_{S}$ is the thickness of the YBCO film, $d_{S R O}$ is the thickness of the SRO film, $d_{L S M O}$ is the thickness of the LSMO film, and $\Delta m$ is the change to the magnetic moment. For heterostructures No. 1 and No. $4 \Delta m$ was measured for the entire sample, whereas for No. 2 and No. 3 it was measured only for the ferromagnetic part. For heterostructure No. $4, \mathrm{Y}_{0.7} \mathrm{Ca}_{0.3} \mathrm{Ba}_{2} \mathrm{Cu}_{3} \mathrm{O}_{x}$ was used as a superconductor. In heterostructures No. 5 and No. 6 the superconductor was not sputtered.

\begin{tabular}{lccccc}
\hline \hline No. & Substrate & $d_{S}(\mathrm{~nm})$ & $d_{\text {SRO }}(\mathrm{nm})$ & $d_{L S M O}(\mathrm{~nm})$ & $\Delta m\left(10^{-6} \mathrm{~nm}\right)$ \\
\hline 1 & $(001) \mathrm{LaAlO}_{3}$ & 80 & 20 & 14 & 10 \\
2 & $(110) \mathrm{NdGaO}_{3}$ & 80 & 17 & 7 & $5 \pm 1.5$ \\
3 & $(110) \mathrm{NdGaO}_{3}$ & 180 & 0 & 20 & $\leq(1 \pm 2)$ \\
4 & $(001) \mathrm{LSAT}$ & 150 & 13 & 25 & 2.5 \\
5 & $(110) \mathrm{NdGaO}_{3}$ & 0 & 14 & 40 & $\ldots$ \\
6 & $(110) \mathrm{NdGaO}_{3}$ & 0 & 0 & 50 & $\cdots$ \\
\hline \hline
\end{tabular}


(polarization of $99.99 \%$ ) fell on the sample at a grazing angle $\theta=(0.15-1)^{\circ}$. The beam divergence $\Delta \theta_{1}=0.025^{\circ}$ was set by two diaphragms before the sample. The polarization of the reflected beam was analyzed using an analyzer with $98 \%$ efficiency. The external magnetic field in the experiment was directed in the sample plane and normal to the scattering plane. At a fixed temperature we took data from 4 channels of small-angle reflection intensity: $R^{++}(\theta), R^{-}(\theta), R^{+-}(\theta)$, $R^{-+}(\theta)$. The "+" and "-" signs should be understood as signs of the neutron spin projection on the external magnetic field. The reflection coefficients without spin flip $R^{++}(\theta), R^{-}$ $(\theta)$ are sensitive to the sum and difference, respectively, of the nuclear profile (SLD) and components of magnetization lying in the plane of the substrate and collinear to the external field $\left(M_{\|}\right)$. The reflection coefficients with the spin flip $R^{+-}(\theta)$, $R^{-+}(\theta)$ are sensitive to the magnetization component $M_{\perp}$, lying in the plane of the substrate and non-collinear to the external field. A feature of the reflectometry of polarized neutrons is insensitivity of the method to the magnetization component, normal to the plane of the sample. ${ }^{47,48}$

We investigated mesastructures in which an $\mathrm{Au}-\mathrm{Nb}$ bilayer served as the top superconducting electrode and was deposited by magnetron sputtering. ${ }^{43}$ The use of an epitaxial film made of a cuprate YBCO superconductor as a bottom electrode is made necessary by the need to ensure epitaxial growth of the oxide interlayer, which is composed of two ferromagnetic materials: LSMO with a uniaxial magnetic anisotropy of 20-30 mT and exchange energy of $2.3 \mathrm{meV} ;{ }^{49}$ and SRO with a magnetic anisotropy of about $1 \mathrm{~T}$ and exchange energy of $13 \mathrm{meV} .{ }^{50}$ Five square-shaped mesastructures with linear dimensions in the plane $L=10,20,30$, 40 , and $50 \mu \mathrm{m}$ are prepared on the substrate (hereinafter referred to as "chip") using photolithography techniques, ion beam etching, and explosive photolithography. An $\mathrm{SiO}_{2}$ film with a thickness of $40 \mathrm{~nm}$ was used to isolate the contact at the ends of the mesastructure. A diagram of the measurements and the cross-section of the mesastructure are shown in Fig. 1(a). The surface properties were tested for filmsatellites on an atomic-force microscope, and fairly sharp boundaries between the SRO/LSMO layers were observed on a transmission electron microscope (Fig. 1(b)).

As shown by transmission electron microscope experiments, the SRO/LSMO boundary in our mesastructures is fairly homogeneous. It is clearly visible on the scale used for the diagram (see Fig. 1(b)). The resistive characteristics of the film-satellites and current-voltage characteristics (CVC) of the mesastructures were measured using a four point
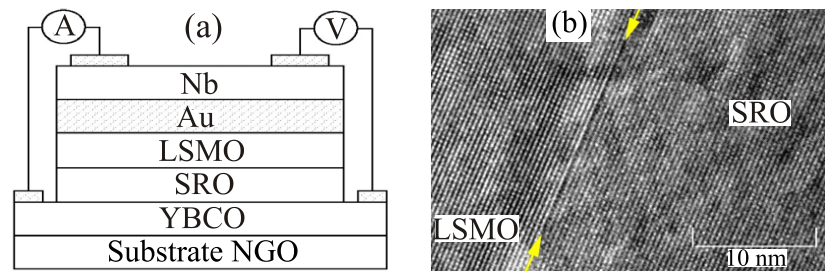

Fig. 1. The cross section and measurement circuit of the mesastructure's electrophysical parameters. A is the current source through the mesastructure and $\mathrm{V}$ is the voltage measuring device (a). An image of the interface between SRO and LSMO, obtained using a transmission electron microscope JEM-2100 with $8 \times 10^{5}$ zoom. The interface location is marked by arrows (b). probe (see Fig. 1(a)) over the temperature range $4.2 \mathrm{~K}<T$ $<300 \mathrm{~K}$, magnetic fields $H$ of up to $2 \mathrm{kOe}$ and microwave exposure of monochromatic radiation at frequencies $f_{e} 1-3$ $\mathrm{GHz}$ and 36-45 GHz. Radiation with a frequency of 1-3 $\mathrm{GHz}$ was applied to the sample with the help of a coaxial cable through separation vessels and the radiation of $\mathrm{mm}$ waves through a waveguide. To reduce the influence of external electromagnetic fields the measurements were conducted in a shielded box using a signal filtration system.

\section{Magnetic measurements of the heterostructures}

Table 1 shows the compositions and thicknesses of the test heterostructure films, as well as the experimentally determined changes to the magnetic moments of the heterostructure, $\Delta m$. The values of $\Delta m$ for samples N2 and N3 are obtained from the FMR measurements and correspond to the changes in magnetization of the SRO layer for heterostructures No. 2 and the LSMO film for heterostructure No. 3; the rest of the measurements pertain to changes in magnetization of the entire structure. In sample No. 4 we used a LSAT substrate, onto which we deposited an epitaxial film made out of a calcium-doped cuprate superconductor $\mathrm{YBCO}\left(\mathrm{Y}_{0.7} \mathrm{Ca}_{0.3} \mathrm{Ba}_{2} \mathrm{Cu}_{3} \mathrm{O}_{x}\right)$.

Fig. 2 shows a family of temperature dependences for the magnetic moment parallel to the substrate plane $m_{\|}$, for
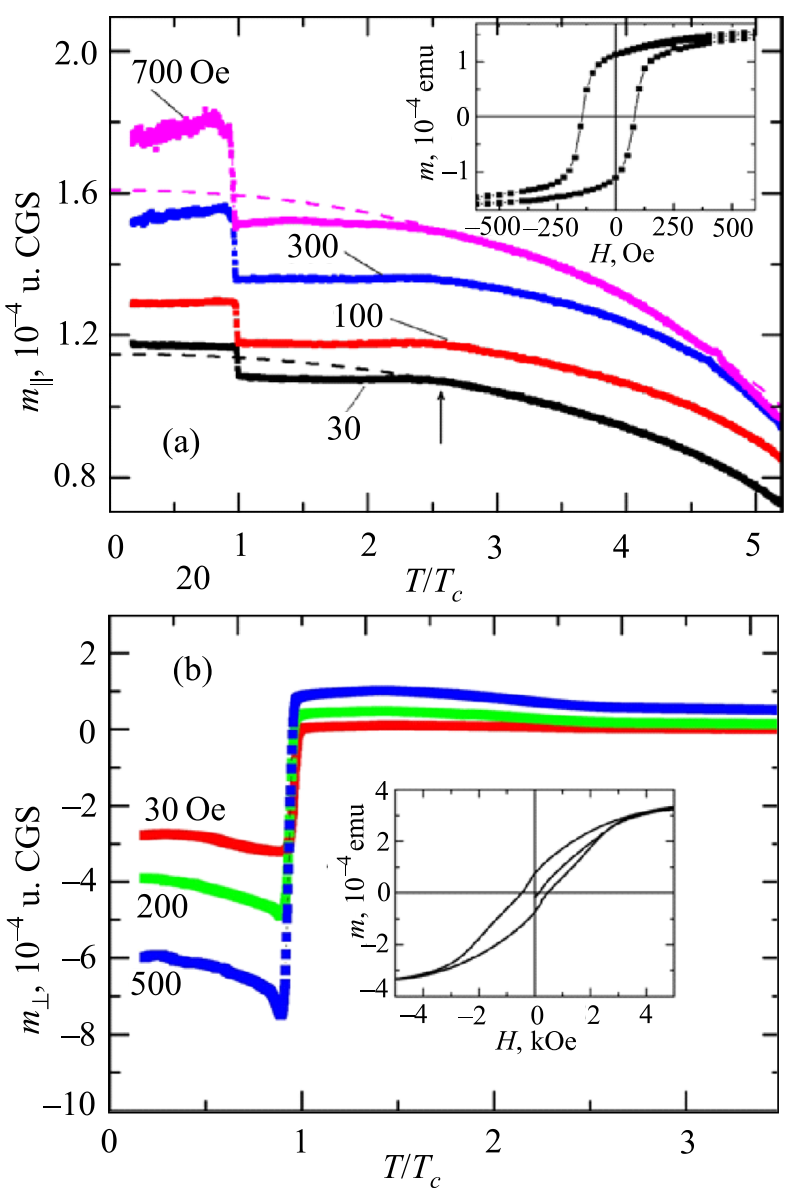

Fig. 2. The temperature dependences of the magnetic moment $m_{\|}$of the $\mathrm{Au} /$ LSMO/SRO/YBCO heterostructure for a magnetic field parallel to the substrate plane, during cooling in a magnetic field $1 \mathrm{kOe}$ (FC mode). The transition of the SRO film into the ferromagnetic state is observed at $T / T_{c}=2.5$. The inset shows the hysteresis loop of the heterostructure for this orientation of the field at $T=100 \mathrm{~K}$ (a). The dependences of the magnetic moment $m_{\perp}$ in a magnetic field perpendicular to the substrate plane. The inset shows the hysteresis loop for this direction of the magnetic filed (b). 
heterostructure No. 1. These dependences are obtained using the SQUID magnetometer during cooling in a magnetic field (FC mode). The external magnetic field was located in the substrate plane and was directed along one of its edges. Detailed measurements of the magnetic anisotropy have shown that the substrate edges form an angle of $40^{\circ}-50^{\circ}$ relative to the easy axis of the LSMO magnetic anisotropy. In the temperature interval $T<T_{S R O}$ (the superconducting transition temperature of the $\mathrm{YBCO}$ film $T_{c} \approx 55 \mathrm{~K}$ and the Curie temperature of the SRO film $T_{S R O} \approx 150 \mathrm{~K}$ for the given heterostructure) $m_{\|}$is determined by the sum of the projections of the LSMO film magnetic moment and the SRO film magnetic moment in the direction of the magnetic field. Under the influence of a magnetic field $m_{\|}$changes due to the rotation of the LSMO and SRO film magnetic moments. As a result the interaction of the LSMO and SRO film magnetic moment the total magnetic moment of the spin valve at low fields is less than that of the LSMO film at the same temperature, whereas at $H>1 \mathrm{kOe}$ it is more.

The results of measuring the field dependence of the $\mathrm{Au} /$ LSMO/SRO/YBCO heterostructure magnetic moment $m$ (see No. 1 in Table 1) are shown in the inset of Fig. 2(a) for a magnetic field directed along the substrate edge at a temperature $T=100 \mathrm{~K}$, which is higher than the critical temperature of the superconductor, $T_{c}$. The position of the magnetization easy axis of the SRO film is close to the normal of the substrate plane. The noncollinearity of the magnetization vectors of the ferromagnetic films contributes to the generation of superconducting triplet correlations having a nonzero spin projection of superconducting carriers, in the ferromagnetic interlayer. $^{43,51,52}$

At $T \approx T_{c}$, for a field parallel to the substrate plane, there is a sharp increase in the magnetic moment of the heterostructure (Fig. 2(a)). The thickness of the YBCO film $d_{S}=80 \mathrm{~nm}$ is less than the London penetration depth of the magnetic field. The magnetic field that is directed along the plane of the film completely penetrates the superconductor and the diamagnetic response is not observed due to the Meissner effect.

The occurrence of a magnetic moment in a cuprate superconductor bordering manganite was theoretically considered in Ref. 42, wherein it was demonstrated that as a result of the antiferromagnetic interaction of the spins $x^{2}-y^{2}$ of $\mathrm{Cu}$ electrons with $e_{g}$-electrons of $\mathrm{Mn}$ there is an induced negative spin polarization in the cuprate superconductor. The impact of this process on the properties of the superconductor is much stronger than injecting the spin-polarized electrons of the ferromagnet. An important parameter in analyzing the ongoing processes is the magnetic moment penetration depth into the superconductor, which is not limited by the changes to the position of the atoms on the neighboring layers of the border, but is determined instead by $8-10$ atomic layers of the superconductor. ${ }^{40,42}$ It was determined in Ref. 17 that for this mechanism of an induced magnetic moment in the $[\mathrm{YBCO} / \mathrm{LCMO}]_{n}$ superlattice, the magnetic moment of the $\mathrm{Cu}$ atom induced in the superconductor is equal to $0.23 \mu_{B}$ and is directed against the magnetic moment of $\mathrm{Mn}$.

Assuming that the change in the magnetic moment of our heterostructure occurs due to copper atoms located in a layer that is about $10 \mathrm{~nm}$ thick, in order to experimentally obtain the value $\Delta m \sim 10^{-5}$ u CGS in sample No. 1 (see Fig. 2(a)) we should take the value of the induced magnetic moment $\sim 0.25 \mu_{B} / \mathrm{Cu}$. The change to the direction of the copper magnetic moment in the YBCO/SRO contacts, observed both in Ref. 53 and just like in our case, can be caused by the negative magnetization of the SRO film. ${ }^{27,45}$

If the magnetic field is directed perpendicularly to the plane of the superconducting film, then the shielding currents occur in the layer $\lambda_{\perp}=\lambda^{2}{ }_{L} / d_{S} \approx 0.3 \mu \mathrm{m}$ on the edge of the film, and the magnetic field gets pushed out of the superconducting film; a diamagnetic response is observed (Meissner effect). This can be seen on the dependence of the magnetic moment $m_{\perp}(T)$ of the heterostructure, measured in the direction of the magnetic field that is perpendicular to the plane of the substrate (see Fig. 2(b)). It is easy to determine the superconductor critical temperature $T_{c}$ in the heterostructure using the dependence $m_{\perp}(T)$. Note that the form of the dependences of perpendicular $m_{\perp}(T)$ does not change if we change modes (FC or ZFC).

\section{Ferromagnetic resonance in the heterostructure}

When measuring the ferromagnetic resonance spectrum the magnetic component of the microwave field was perpendicular to the plane of the substrate. The external magnetic field $H$ was always located in the plane of the substrate (parallel orientation) whereas in experiments with sample cooling it was put along the magnetization easy axis of the induced uniaxial anisotropy of the LSMO film. The direction of this axis was pre-determined from the angular dependences of the resonance field $H_{C F}$, taken at different temperatures under the conditions of magnetic field rotations around the normal to the substrate plane in a parallel orientation. ${ }^{44}$

The angular dependences of the FMR spectrum of thin ferromagnetic film in the presence of uniaxial and biaxial anisotropy are described by the following equation: ${ }^{44}$

$$
\begin{aligned}
\left(\frac{\omega}{\gamma}\right)^{2}= & \left(H_{0}+H_{u} \cos 2 \varphi_{u}+H_{c} \cos 4 \varphi_{c}\right) \\
& \times\left(4 \pi M_{0}+H_{0}+H_{u} \cos ^{2} \varphi_{u}+H_{c} \frac{1+\cos ^{2} 2 \varphi_{c}}{2}\right),
\end{aligned}
$$

wherein $(\omega / \gamma)$ is the gyromagnetic ratio, $H_{u}=2 K_{u} / M_{0}$, $H_{c}=2 K_{c} / M_{0}, K_{u}$ is the uniaxial anisotropy constant, $K_{c}$ is the cubic anisotropy constant, the $M_{0}$ parameter is equal to the equilibrium magnetization in the absence of adjacent magnetically ordered layers, and $\varphi_{u}$ and $\varphi_{c}$ are angles at which the uniaxial and cubic anisotropy easy axes of magnetization are directed, relative to the external magnetic field, respectively. As a result of adjusting the experimental data (Fig. 3) using Equation (1), we were able to determine the following ferromagnet parameters: $K_{u}, K_{c}, M_{0}$, as well as the direction of both the uniaxial and cubic anisotropy easy axes (see inset in Fig. 3).

As noted previously, the processing of the angular dependences of the FMR spectra according to Equation (1) allows us to determine the $M_{0}$ parameter and the directions of the easy axes in the LSMO films of the test heterostructures. The interlayer exchange between two ferromagnets 


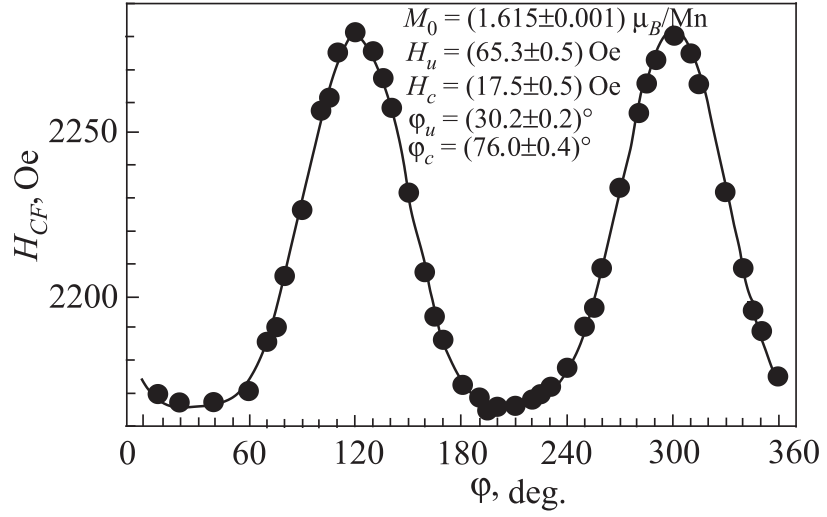

Fig. 3. The angular dependence of the resonant magnetic field at $T=295 \mathrm{~K}$, heterostructure $\mathrm{Au} / \mathrm{LSMO} / \mathrm{SRO} / \mathrm{YBCO}$. The solid line shows Equation (1) with adjustable parameters, shown in the inset.

must be considered at lower temperatures, because it leads to a resonance relation that differs from Equation (1).

Fig. 4 shows the FMR spectra of the LSMO film from heterostructure No. 2, taken at $T \approx T_{c}$. At $T \leq T_{c}$, when the YBCO film transitions to the superconducting state, there is a huge signal of nonresonant absorption at low magnetic fields having a hysteresis in the magnetic field. As a result the FMR signals at $T<T_{c}$ are recorded against a backdrop of a giant non-resonant absorption, which increases the error in the measurements of the resonant field $H_{C F}$, but does allow us to determine the superconducting transition temperature $T_{c}$ of YBCO films. At $T>T_{c}$ the values of $H_{C F}$ are determined much more accurately, as shown by the inset on Fig. 4.

Fig. 5 shows the temperature dependences of the resonant field $H_{C F}$ for FMR signals from LSMO films in $\mathrm{Au} /$ $\mathrm{LSMO} / \mathrm{SRO} / \mathrm{YBCO}$ and $\mathrm{Au} / \mathrm{LSMO} / \mathrm{YBCO}$ heterostructures in the superconducting transition range of the YBCO films. In all cases the direction of the external magnetic field was taken along the easy magnetization axis. It is evident that for the $\mathrm{Au} / \mathrm{LSMO} / \mathrm{SRO} / \mathrm{YBCO}$ heterostructure there is a sharp change in the resonant field in the superconducting transition range.

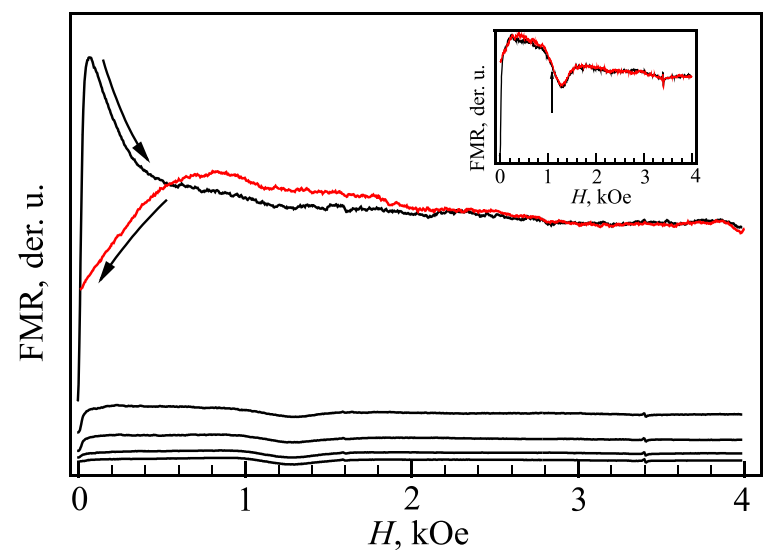

Fig. 4. Temperature dependences of the FMR spectra of the Au/LSMO/ $\mathrm{SRO} / \mathrm{YBCO}$ heterostructure at temperatures (top to bottom): $4 \%, 3 \%, 2 \%$, and $1 \%$ higher than $T_{c}$, as well as at $T<T_{c}$. The arrows indicate the direction of the changes in the magnetic field during the measurement of the absorption spectrum at $T<T_{c}$. The inset shows the FMR spectrum (direct and reverse course with respect to the field) for $T=1.01 T_{c}$, the arrows indicate the resonant field $H_{C F}$.

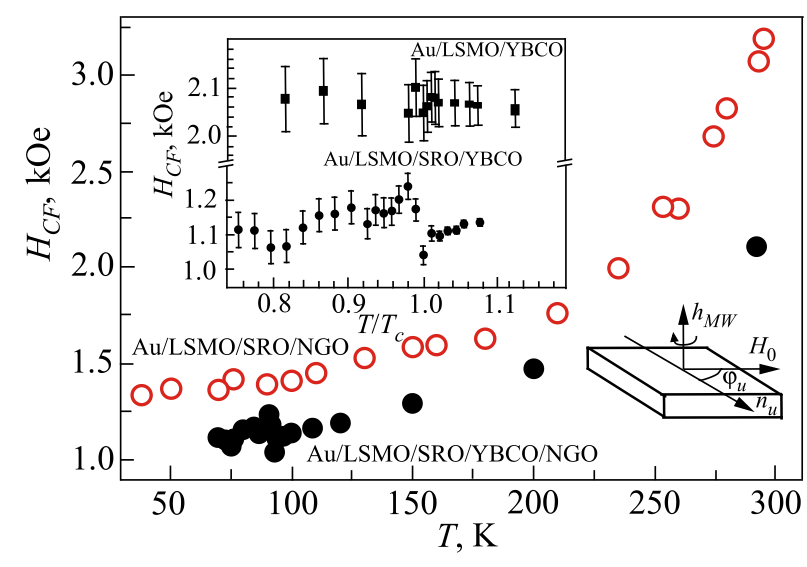

Fig. 5. The temperature dependence of the resonance field in the LSMO film for two heterostructures: Au/LSMO/YBCO and Au/LSMO/SRO/YBCO. The top inset shows the temperature dependence of the resonance field of these heterostructures in the vicinity of $T_{c}$. The bottom inset shows the geometry of the FMR spectrum measurements.

Since in the $\mathrm{Au} / \mathrm{LSMO} / \mathrm{SRO} / \mathrm{YBCO}$ heterostructure the LSMO film, in which changes to the resonance field are recorded, is separated from the superconducting YBCO film by the ferromagnetic SRO film, the jump of the resonance field HCF of the LSMO layer should be associated with the change in magnetization in the SRO film that is in direct contact with the superconducting layer. To do this, one must take into account the interlayer exchange interaction between LSMO and SRO, which occurs through the magnetically ordered boundary layer with a high conductivity. ${ }^{54-57}$ Using the procedure outlined in Refs. 58 and 59, we obtained an expression that describes the relationship between the frequency and the resonance field for the LSMO layer in the LSMO/SRO heterostructure; the expression is similar to Equation (1) but its value for the resonant field should be replaced with this combination

$$
H_{C F}+\frac{H_{J 1}^{L S M O}\left(H_{C F}+H_{J 1}^{S R O}\right)}{H_{S R O}-4 \pi M_{S R O}-H_{J 2}^{S R O}} .
$$

Here $H_{S R O}$ and $M_{S R O}$ represent the field of the uniaxial magnetic anisotropy and the magnetization of the SRO film, $H_{J 1}^{L S M O, S R O}$ and $H_{J 2}^{S R O}$ are the effective fields of bilinear and biquadratic interlayer exchanges for the corresponding layers, and the magnitudes of these fields are inversely proportional to the magnetization of the corresponding layers. ${ }^{58,59}$

Observance of the resonance ratio requires that the combination in Equation (2) be constant on both sides of the magnetization jump. It is based on this condition of constancy that we obtain the relation between the jump of the resonant field $\delta H_{C F}$ in the LMSO film and the magnetization jump of the SRO film $\delta \mathrm{M}_{S R O}$ :

$$
\frac{\delta M_{S R O}}{M_{S R O}} \approx \frac{\delta H_{C F}}{H_{C F}} \frac{H_{S R O}}{4 \pi M_{S R O}} .
$$

An assessment performed in accordance with Equation (3) shows that change to the magnetization of the SRO film during the YBCO transition to the superconducting state is about $\sim 0.5 M_{S R O}$. Taking into account the contribution of the SRO film $\left(m_{S R O} \sim 10^{-5}\right.$ u CGS $)$ to the total magnetic moment $m_{\|}$of the heterostructure (Fig. 2(a)) we find that the 

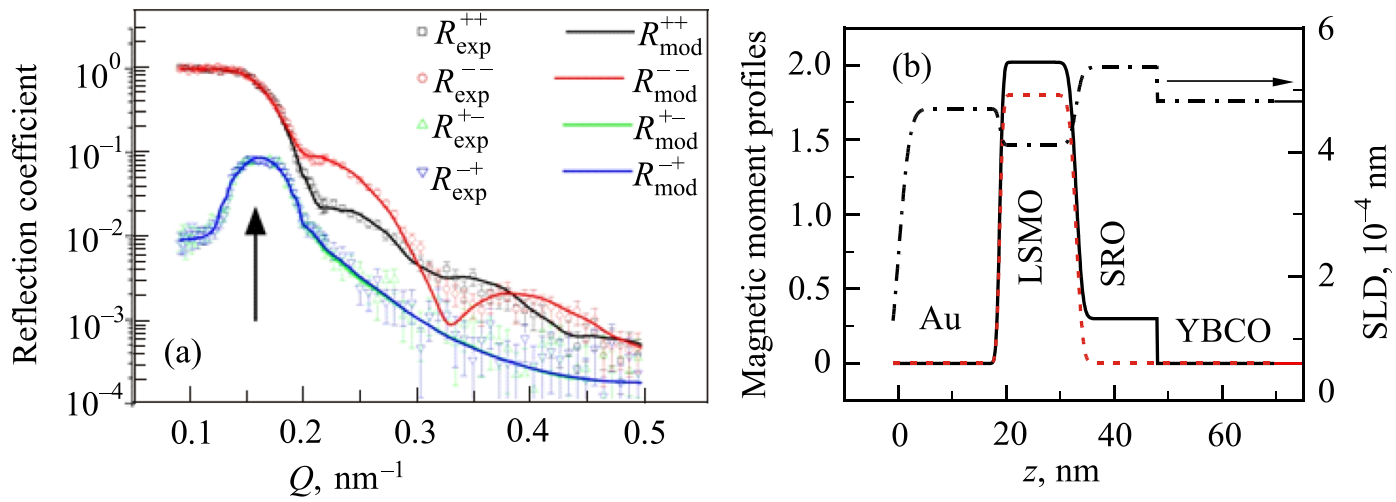

Fig. 6. The reflection coefficients of low-energy neutrons from the Au/LSMO/SRO/YBCO heterostructure at $T=80 \mathrm{~K}, H=30$ Oe. The calculated reflection coefficient curves are shown by solid lines. The calculated dependences for reflection coefficients with spin flip $R^{+-}(\theta)$ and $R^{-+}(\theta)$ coincide. The arrow indicates the position of the waveguide peak that coincides with the critical value of the transmitted torque of total reflection $Q_{\text {crit }}$ (a). The nuclear SLD profile (dot dash) and the profile of the heterostructure's magnetic moments in the plane of the substrate: $m_{\|}$(solid line) and $m_{\perp}$ (dotted line) (b).

change to the magnetic moment of the composite ferromagnet is less than the magnetic moment induced in the superconductor. Note that the positive sign $\delta M_{S R O}$ indicates that the magnetization of the SRO film decreases, since in this layer the magnetization has a negative sign (see also Ref. 27).

According to Fig. 5, we can also see that in the $\mathrm{Au} /$ LSMO/YBCO heterostructure, in which the ferromagnetic LSMO film comes into contact with the YBCO film, a remarkable change in the magnetization of LSMO at $T \approx T_{c}$, is not detected, within error. This difference in the Au/LSMO/ YBCO heterostructure can be explained by the absence of an excitation of the triplet component of the superconducting current in the ferromagnetic interlayer ${ }^{22,27,51,52,60}$ and low transparency of the YBCO/LSMO border. ${ }^{52}$ This leads to a negligibly small penetration of the superconducting order parameter from YBCO into the LSMO film and therefore, to a negligibly small change in the magnetic moment of the LSMO film in the heterostructure.

\section{Neutron measurements}

Neutron-reflectometry curves were measured for $\mathrm{Au} /$ LSMO/SRO/YBCO heterostructures over temperatures ranging from $T=10-80 \mathrm{~K}$. The experiment showed an increase in spin-flip scattering (SF scattering) at temperatures below $T_{c}$. However, SF scattering at temperatures above and below $T_{c}$ did not change within statistical error. Fig. 6(a) shows the reflection coefficients measured at $T>T_{c}$ for the Au/LSMO/ YBCO heterostructure. The reflection coefficients without a reversal (non-spin flip, NSF scattering) $R^{++}(\theta)$ and $R^{--}(\theta)$ are characterized by a region of total reflection when $Q<Q_{\text {crit }} \approx 0.15 \mathrm{~nm}^{-1}$ and Kissig oscillations, caused by interference at the interfaces of the structure. Essential differences between the NSF reflection coefficients $R^{++}(\theta)$ and $R^{--}(\theta)$ indicates that a collinear magnetization component is present. At the same time, strong SF scattering was observed in the experiment, which indicates that there is also a non-collinear magnetization component. The presence of a resonance peak near $Q_{\text {crit }}$ is typical for SF scattering in this structure, and this peak is caused by a resonance-enhanced neutron standing wave. ${ }^{47,48,61}$

Adjusting the experimental data for the model proposed in Ref. 51 allowed us to restore the nuclear scattering length density (SLD) profile, as well as the profile of the collinear and non-collinear components of the magnetic moment in the plane (Fig. 6(b)). As follows from the adjustment, the interfaces in the structure are fairly sharp with transition regions that do not exceed $2 \mathrm{~nm}$. The magnetic state of the system is well described by the magnetization of the LSMO film $2.5 \mu_{B} / \mathrm{Mn}$, directed at an angle of $43.3^{\circ}$ to the external field, and the SRO component of magnetization in the plane with a magnitude of $0.3 \mu_{B} / \mathrm{Ru}$, parallel to the external field. SF scattering above $T_{c}$ is given by the magnetization component of the LSMO layer, which is normal to the external field.

Fig. 7 shows the SF scattering intensity in the vicinity of the resonance peak, and is measured at temperatures above and below $T_{c}$. As shown in Fig. 7, the intensity of the SF scattering increased as the sample transitioned into the superconducting state. The inset of Fig. 7 shows the temperature dependence of the waveguide peak intensity, from which the growth of the SF scattering follows, at temperatures below $T_{c}$.

For the Au/LSMO/SRO/YBCO heterostructure the performed calculation shows that the growth of SF scattering can be described sufficiently well by the appearance of magnetization in the YBCO layer with a magnitude of $0.4 \mu_{B} / \mathrm{Cu}$ at a thickness of $10 \mathrm{~nm}$ in the vicinity of the border with

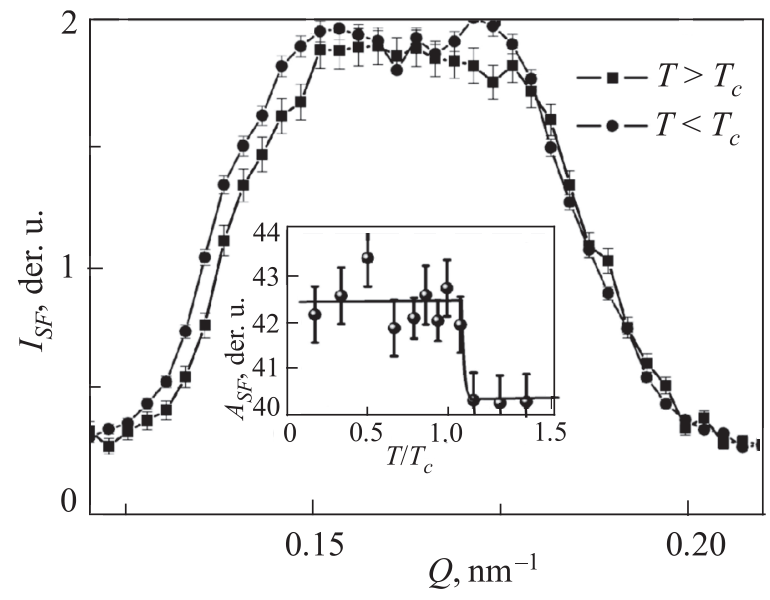

Fig. 7. The temperature dependence of the waveguide peak intensity $I_{S F}$ for the $\mathrm{Au} / \mathrm{LSMO} / \mathrm{SRO} / \mathrm{YBCP}$ heterostructure. The lines connect the data points. The inset shows the temperature change in the area of the waveguide peak. 
SRO. At the same time the vector of the induced magnetization must be parallel to the magnetization of the LSMO layer, i.e., at an angle of $45^{\circ}$ to the external field. Moreover, changes to scattering without spin flip (NSF scattering) (about 1\%-3\%) due to this torque, does not exceed the statistical error of experimentally measured reflection coefficients. Another model describing the growth of SF scattering involves a magnetic moment with a magnitude of $4 \mu_{B} / \mathrm{Cu}$ at a thickness of $1 \mathrm{~nm}$ (of about $\xi_{S}$ ) near the boundary with SRO, which causes a strong change in NSF scattering coefficients (more than 10\%), which is not observed in the experiment.

Models suggesting the growth of non-collinear magnetization in the LSMO or SRO films also describe the experimental data, but are contrary to the FMR data according to which the magnetization of the ferromagnetic interlayer should only decrease.

\section{Electron transport in mesastructures}

Micron-sized mesastructures, in which the two superconductors $\mathrm{YBCO}$ and the $\mathrm{Au}-\mathrm{Nb}$ bilayer are separated by a magnetic spin valve SRO/SLMO were used to determine the penetration depth of superconducting correlations into the ferromagnet. By varying the thickness of the interlayer, it was possible to estimate the penetration depth of superconducting correlations into the ferromagnetic layer.

On the curves showing the electrical resistance $R$ of the mesastructures as a function of temperature (Fig. 8(a)), there are two highlighted regions of resistance reduction, which correspond to the transition of $\mathrm{YBCO}$ and $\mathrm{Au}-\mathrm{Nb}$ films into the superconducting state. Above the critical temperature YBCO $T_{c}^{Y B C O}$ the dependence $R(T)$ has a linear metal course, which is typical for the temperature dependence of a YBCO electrode (see Fig. 8(b)). At $T<T_{c}^{Y B C O}$ the value of $R$ decreases rapidly (Fig. 8(a)), while the features that are typical for the temperature dependences (Fig. 8(b)) of autonomous films included in the mesastructure, are not observed in this temperature range. This behavior is explained by the fact that below the critical temperature of YBCO the contribution from LSMO and SRO films into the value $R_{N} A$ (area $A=L^{2}$ ) is inferior to the contribution of the boundary resistance at the mesastructure film interfaces. As shown by the additional measurements, the resistance of the two-layer $\mathrm{Au}-\mathrm{Nb}$ film is also small. ${ }^{62}$ As a result, in the temperature range $T<T_{c}^{Y B C O}$ the resistance of the mesastructure is combined with the resistance of the interfaces between the boundaries between materials YBCO/SRO, SRO/LSMO,LSMO/Au: $R_{M S}=R_{Y B C O / S R O}$ $+R_{S R O / L S M O}+R_{L S M O / \mathrm{Au}}$.

In order to clarify the contribution from each of the interfaces that define the resistance of the composite layer mesastructure, we also prepared mesastructures with a single ferromagnetic interlayer. For structures with an SRO interlayer the value $R_{N} A$ is almost three orders of magnitude lower than for a structure with an LSMO interlayer. If we assume that the resistance of the LSMO/Au border does not exceed the value of $1 \mu \Omega \mathrm{cm}^{2}$ (Ref. 63) then the resistance of the YBCO/LSMO/Au mesastructure $\left(100 \mu \Omega \mathrm{cm}^{2}\right)$ can be explained by the greater resistance of the YBCO/LSMO interface. Using the data from Ref. 63 we find that the resistance of the SRO/Au interface can be estimated to be $0.05 \mu \Omega \mathrm{cm}^{2}$, whereas the resistance of the YBCO/SRO border is about $0.1 \mu \Omega \mathrm{cm}^{2}$, which is consistent with the data in Ref. 64. Consequently, the value of $R_{N} A$ of the mesastructure is determined mainly by the sum of the resistances of $R_{L S M O} /$ $\mathrm{Au}$ and $R_{Y B C O / S R O}{ }^{52}$

\section{The critical current of a mesastructure}

A critical current is observed in most of the measured mesastructures with a composite interlayer thickness of $50 \mathrm{~nm}$. The current decreases linearly as the temperature increases (see inset in Fig. 8(a)) over the temperatures $4.2 \mathrm{~K}$ $<T<T_{c}^{\mathrm{Au}-\mathrm{Nb}}$. For comparison, in mesastructures with one ferromagnetic interlayer (LSMO or SRO) the superconducting current is absent at interlayer thicknesses exceeding $5 \mathrm{~nm}$, which is about equal to the coherence length $\xi_{F}$, an estimate of which will be provided below. At smaller interlayer thicknesses the superconducting current found on some samples was caused by current flow through short circuits.

The presence of a critical current and its exponential decline at spin valve thicknesses greater than $5 \mathrm{~nm}$ is an indication of the spin-triplet superconducting correlation transport via the spin valve. ${ }^{13,65}$ Outlines of the experimental
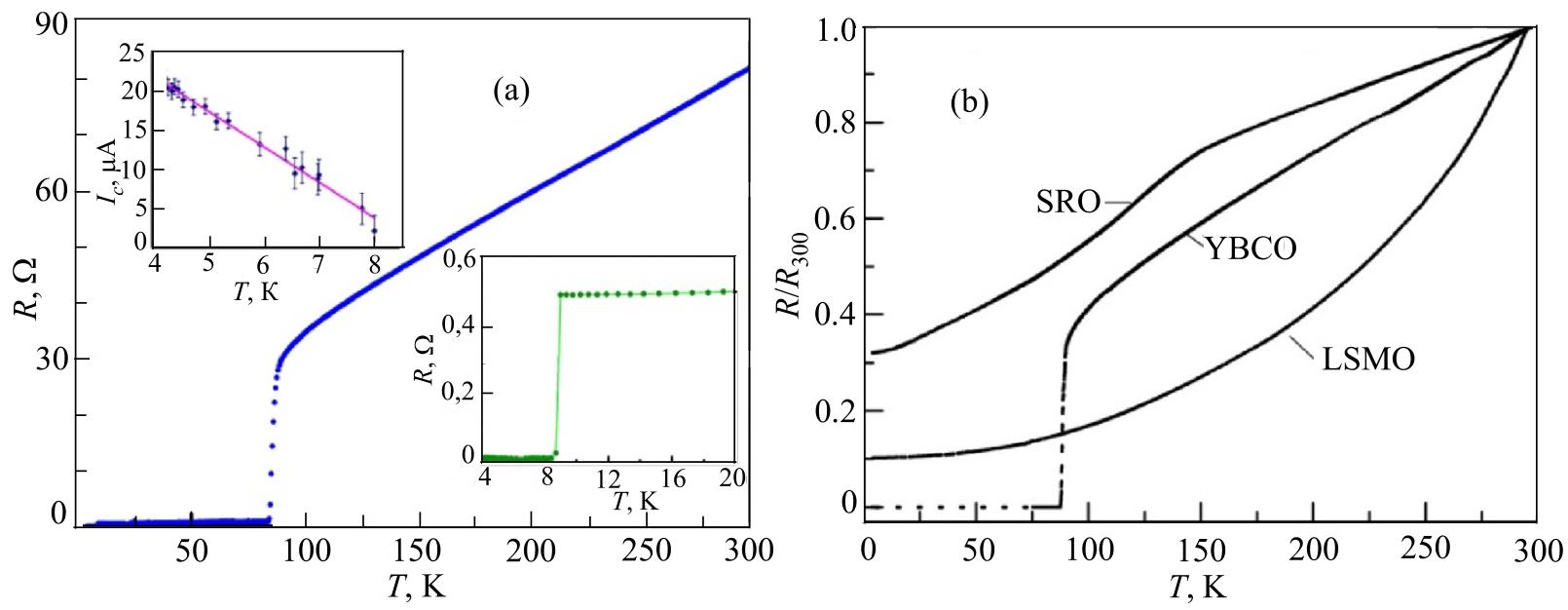

Fig. 8. The temperature dependence of the mesastructure resistance $R(T)$. The bottom panel shows an enlarged area of $R(T)$ at low temperatures, whereas the top panel shows the temperature dependence of the critical current (a). The temperature dependences of the normalized resistance of YBCO, SRO and LSMO films with a thickness of 100,60, and $55 \mathrm{~nm}$, respectively, deposited directly on to a (110)NGO substrate (b). 


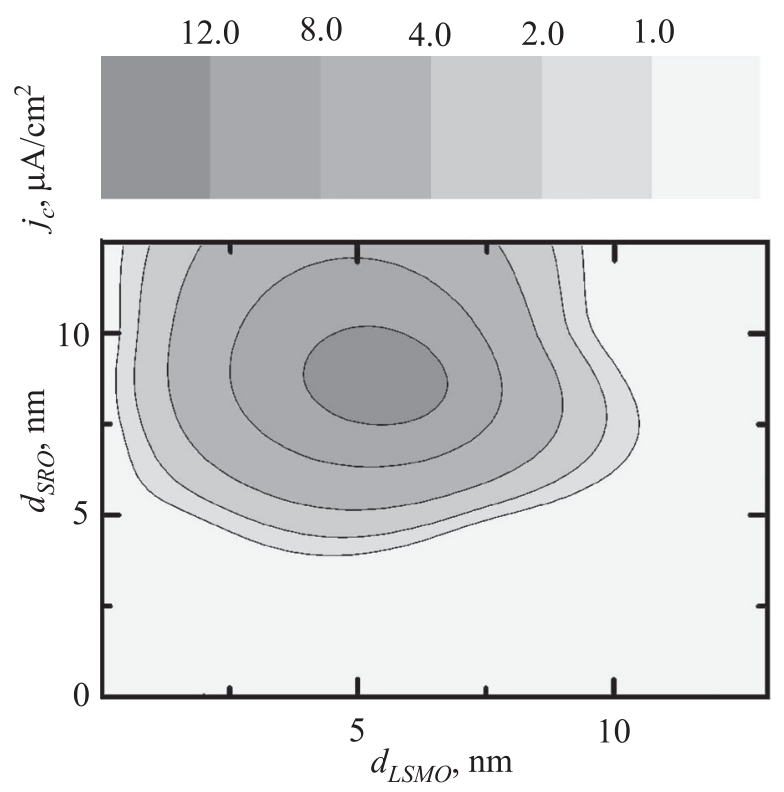

Fig. 9. Outlined regions of critical current density as a function of the plane thickness of the spin valve ferromagnetic layers at $T=4.2 \mathrm{~K}$.

values for the critical current density $j_{c}$ for LSMO and SRO film thicknesses between 0 and $20 \mathrm{~nm}$, are shown in Fig. 9. We can see a critical current density peak at layer thicknesses $d_{L S M O} \approx 6 \mathrm{~nm}$ and $d_{S R O} \approx 8 \mathrm{~nm}$. Note that the critical current maximum of the TSC in superconducting structures with a two-layer composite ferromagnetic interlayer is predicted at thicknesses that are about equal to the coherence length. ${ }^{66}$

Since the mean free path $l$ in oxide materials (SRO and LSMO) is sufficiently small, ${ }^{67,68}$ we can assume that the electron transport is diffusive in nature. By using the measured dependences $R(T)$ for SRO and LSMO films, we get $\xi_{F}^{L S M O} \approx 8 \mathrm{~nm}$ and $\xi_{F}^{S R O} \approx 3 \mathrm{~nm}$, respectively. Fig. 9 shows that the critical current maximum is observed at $d_{L S M O}$ that are slightly less than our estimate of $\xi_{F}^{L S M O}$, whereas for the SRO layer it is the other way around and observed at values larger than the coherence length $\xi_{F}^{S R O}$.

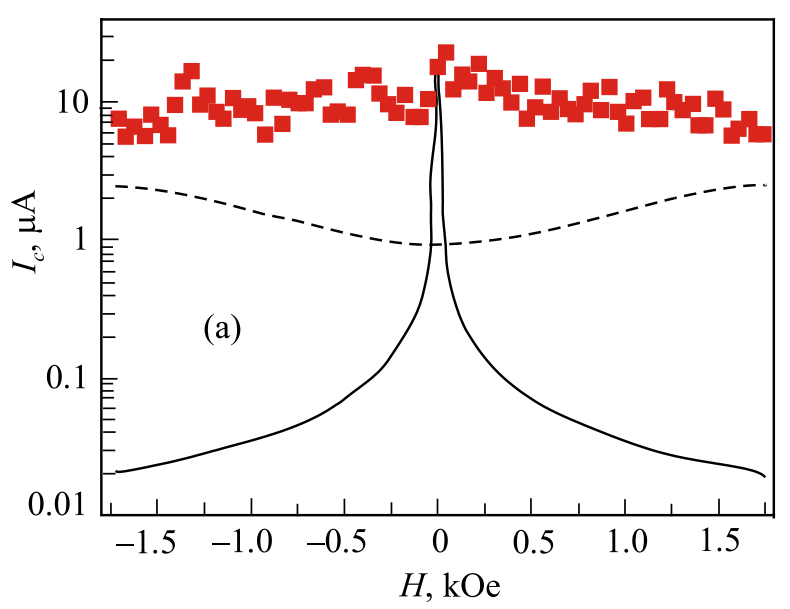

\section{Magneto-field dependences}

In the case of a Josephson junction with a uniform critical current distribution, the critical current as a function of the magnetic field parallel to the junction plane is described by the Fraunhofer relationship

$$
I_{c}(H)=I_{c}(0)\left|\frac{\sin \left(\pi \Phi / \Phi_{0}\right)}{\pi \Phi / \Phi_{0}}\right|
$$

wherein $\Phi_{0}=2.06783461 \times 10^{-7} \mathrm{G} \mathrm{cm}^{2}$ is the magnetic flux quantum and $\Phi=\mu_{0} H S_{\text {eff }}$ is the magnetic flux of the external field in the mesastructure. ${ }^{69,70}$ The zeros (minimums) of the Fraunhofer dependence are observed when the external field flows through a cross-section of the mesastructure equal to the magnetic flux quantum $\Phi \approx \Phi_{0}$. The measured magneto-field dependences of the mesastructure critical current were markedly different from the results of Equation (4). When there is a change in the direction of the field (from ascending to descending, and vice versa), hysteresis is observed on a large change of the field changes, caused by the ferromagnetic nature of the interlayer materials. ${ }^{43}$ Moreover, the critical current was observed at considerably high field values, all the way up to $2 \mathrm{kOe}$ in the experiment (see Fig. 10(a)). Therefore, at $H=-1.3 \mathrm{kOe}$ the value $I_{c}=16.5 \mu \mathrm{A}$, which composes $94 \%$ of the $I_{c}(H=0)$ and 0.7 of the maximum measured at $H=-6.5$ Oe. Note that in $\mathrm{YBCO} / \mathrm{Au} / \mathrm{Nb}$ structures without a magnetic interlayer ${ }^{71}$ but with an antiferromagnetic $\mathrm{Ca}_{0.7} \mathrm{Sr}_{0.3} \mathrm{CuO}_{2}$ interlayer $^{72}$ the critical current dropped sharply with an increasing magnetic field, while it increased in the mesastructures with a spin valve at fields greater than $1 \mathrm{kOe}$. This unusual behavior of the critical current in structures with a metallic ferromagnetic interlayer was mentioned din Ref. 73.

There are several mechanisms that determine the critical current magneto-field dependence: the penetration of the magnetic flux quanta (Josephson vortices), which creates the "Fraunhofer" oscillation, the emergence of a domain structure in the ferromagnetic interlayer, and the rotation of the layer magnetization under the influence of a magnetic field. Note that in the absence of TSC the superconducting current,

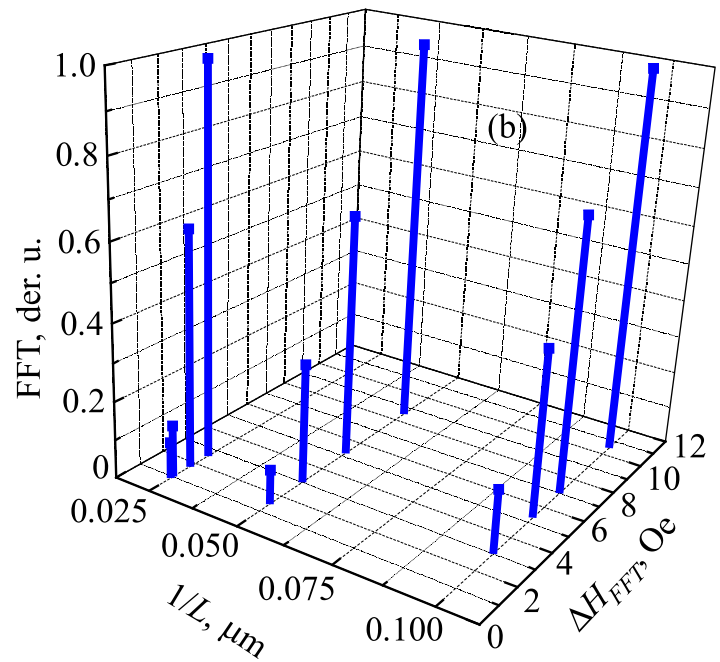

Fig. 10. The dependence of the critical current on the magnetic field over a wide range of magnetic fields for the mesastructure with $d_{S R O}=8.5$ nm, $d_{L S M O}=3 \mathrm{~nm}, L=10 \mathrm{~nm}$. The solid line shows the expected decline of the maxima of the values $I_{C}$ of the Fraunhofer oscillatory dependence (4). The dotted line shows the level of noise that limits our ability to measure the critical current (a). The periods $\Delta H_{F F T}$ and amplitudes of the Fourier components as a function of parameter $1 / L$ for magneto-field dependences of the critical current of 3 mesastructures with $L=10 \mu \mathrm{m}, 20 \mu \mathrm{m}$, and $40 \mu \mathrm{m}$, arranged on a single chip (b). 
according to Equation (4), must be significantly suppressed in the magnetic field at $\Phi \gg \Phi_{0}$, when several quanta of magnetic flux penetrate into the transition, since the maxima of the Fraunhofer dependence fall at a rate of $1 / \Phi$ (see Fig. 10(a)).

In assessing the effective area of penetration of the magnetic field directed along the plane of the transition $S_{\text {eff }}=L d^{\prime}$, the magnetic permeability of the layers must be taken into account $d^{\prime}=\mu_{1} d_{L S M O}+\mu_{2} d_{S R O}+\lambda_{\mathrm{Nb}}+\lambda_{Y B C O}$, wherein $\lambda_{\mathrm{Nb}}=90 \mathrm{~nm}$ and $\lambda_{Y B C O} 150 \mathrm{~nm}$ are the London penetration depths of the magnetic field for $\mathrm{Nb}$ and YBCO, respectively, and $\mu_{1,2}$ is the magnetic permeability. For the Josephson junctions with a ferromagnetic interlayer the effective thickness increases by $\mu=1+\chi$ times, ${ }^{70,74}$ wherein $\chi$ is the magnetic susceptibility. The values $\mu_{1}=12, \mu_{2}=3$ were obtained from the magneto-field dependences of the spin valve interlayer magnetic moment of a mesastructure with $d_{L S M O}=6 \mathrm{~nm}$ and $d_{S R O}=8.5 \mathrm{~nm}$, and $L=10 \mu \mathrm{m}$. When we substitute these values we find that the critical current minima for the mesastructure interlayer should be located at a distance of $\Delta H=6 \mathrm{Oe}$ due to the penetration of the Josephson vortices. This value is slightly different from the experimental value of $\Delta H \approx 10 \mathrm{Oe}$, which is the distance between the minima for $I_{c}(H)$. This difference may be due to the fact that during the assessment of $\chi$ we used data from the measurement of the magnetic moment $M(H)$, which were obtained for a direction of the external magnetic filed coinciding with the hard axis. ${ }^{51}$ Fourier analysis of the oscillatory dependences $I_{c}(H)$, taken on the scale of fields of up to \pm 30 Oe for three mesastructures located on one chip and having an identical thickness $d^{\prime}$, shows the presence of no less than two periods $\Delta H_{F F T}$ with significant FFT amplitudes (see Fig. 10(b)). At the same time, there is an increase in the $\triangle H_{F F T}$ periods proportional to $L^{-1}$.

It is known that the domain structure in the ferromagnetic interlayer could have a dramatic effect on the electron transport mechanism. ${ }^{75,76}$ It is possible that the domaingenerated magnetization non-uniformities in the LSMO films could lead to additional modulations of the $I_{c}(H)$ dependences. However, based on the data in Fig. 10(b) we can see that the oscillations $I_{c}(H)$ are not caused by the domain structure, since in order for this to happen the effective area of the magnetic field penetration $S_{\text {eff }}=d_{\text {dom }} d^{\prime}$ must correspond to much greater periods of critical current oscillations due to the magnetic field, than the values $\Delta H_{F F T}$ in the figure. The presence of the Fourier expansion components with fractional periods $\Delta H_{F F T}$ is most likely indicative of CPD deviation from the sinusoidal form, ${ }^{51,52}$ than of domain structure influence.

\section{Microwave dynamics of mesastructures}

A study of the high-frequency dynamics of Shapiro steps occurring along the $\mathrm{CVC}$ when exposed to microwave radiation proves the absence of direct contact ("short-circuiting") between superconductors. This is confirmed by the presence of Shapiro step oscillations in response to microwave power, the amplitudes of which are in good agreement with the resistively shunted Josephson junction model. ${ }^{62}$ The absence of "short-circuiting" is ensured by a sufficiently thick interlayers, wherein the roughness of the layers is much less than the thickness of LSMO and SRO films.

A comparison of the experimental Shapiro steps with those calculated according to the modified resistive model ${ }^{62}$ allows us to determine the CPD of the mesastructure critical current. Measurement of the current-phase dependence was conducted in a zero magnetic field, and during mesastructure cooling in a constant magnetic field (100-200 Oe), parallel to the substrate plane, starting from a temperature of $160 \mathrm{~K}$.

A family of CVCs obtained under exposure to microwave radiation at a frequency of $41 \mathrm{GHz}$ is shown in Fig. 11(a). Since the frequency of the microwave exposure $f_{e}$ is significantly greater than the characteristic frequency of the mesastructure $f_{e} \gg f_{c}=(2 e / h) I_{c} R_{N}$, then the capacitance effect of the mesastructure could be ignored (McCumber parameter $\left.\beta_{c}=\left(2 \pi / \Phi_{0}\right) I_{c} R^{2 N} C \ll 1\right)$. Under these conditions the appearance of fractional Shapiro steps on the CVC (see Fig. 11(a)) clearly points to the non-sinusoidal nature of the current-phase dependence. ${ }^{62}$ Fig. 11(b) shows the experimental and theoretical dependences of the critical current $I_{c}$ and the first Shapiro step $I_{1}$ on the normalized values of the microwave current $x=I_{R F} / \mathrm{I}_{C} \omega$ wherein $I_{R F}$ is the amplitude
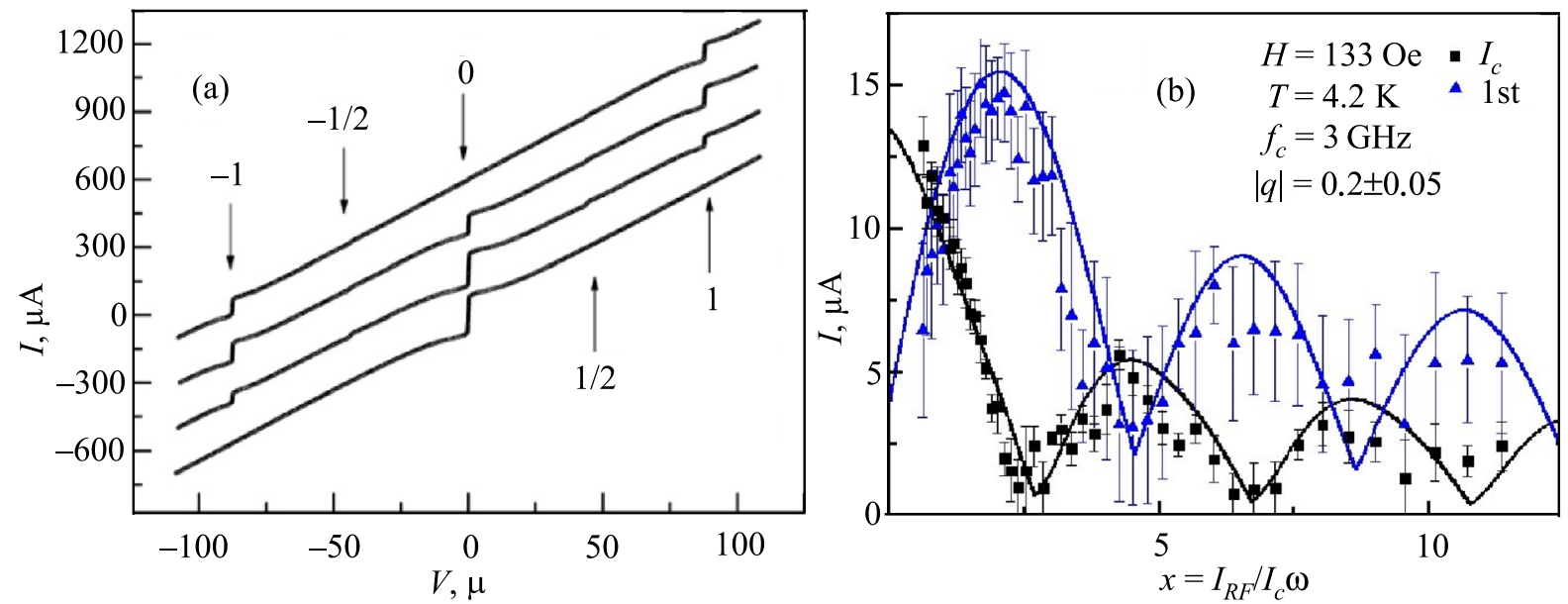

Fig. 11. Family of CVCs for the mesastructure with $d_{S R O}=8.5 \mathrm{~nm}, d_{L S M O}=6 \mathrm{~nm}, L=10 \mu \mathrm{m}$ when subjected to electromagnetic radiation with a frequency $f_{e}=41 \mathrm{GHz}$. The arrows indicate the number $n$ of the Shapiro steps on the voltage axis, $n=0$ corresponds to the critical current $I_{c}$ (a). The dependence of the critical current amplitude and the first Shapiro step for the mesastructure with $d_{S R O}=5.6 \mathrm{~nm}, d_{L S M O}=15 \mathrm{~nm}, L=50 \mu \mathrm{m}$ when subjected to microwave radiation $f_{e}=3 \mathrm{GHz}(\mathrm{b})$. 
of the microwave current $\omega=f_{e} / f_{c}$. The ratio of the amplitude of the CPD second harmonic to the first $q=I_{c 2} / I_{c 1}$ was determined according to the numerical approximation of the critical current and Shapiro step amplitudes as functions of the microwave power. ${ }^{51,52}$ Due to the influence of the CPD second harmonic the critical current and the first Shapiro step have non-zero local minima (see Fig. 11(b)). Since it was assumed that $I_{c 1} \approx I_{c}$ (valid for $q<1$ ), then the values of $q$ determined by this method turn out to be somewhat underestimated. According to the theoretical study in Ref. 13, during the excitation of spin-triplet correlations in a Josephson junction with bilayer ferromagnetism, the second harmonic dominates in CPD, increasing with the disorientation angle of interlayer layer magnetization, reaching a maximum at angles close to $\pi / 2$. According to the measurements obtained using the SQUID-magnetometer, at low fields the magnetization projection of the SRO film is directed in the direction opposite to the magnetization of LSMO. For the LSMO film, the magnetization direction angle with respect to the direction of the external magnetic field is determined by its value, whereas at fields greater than the values of the anisotropy field (200-300 Oe), the magnetization of the LSMO layer is directed along the field. Therefore, in small fields, we should observe the growth of the CPD second harmonic. However, in the microwave experiment on five mesastructures we did not observe an increase in the share of the second harmonic in magnetic fields $20-50$ Oe, as predicted in Refs. 12-14, and its contribution did not exceed the value of $q=0.5$.

\section{Conclusion}

The manifestation of an induced magnetic moment in the superconductor is experimentally observed in a heterostructure based on a cuprate superconductor with a ferromagnetic spin valve. By order of magnitude, the magnetic moment occurring in the superconductor coincides with the calculations for the magnetic moment of the $\mathrm{Cu}$ atoms, induced due to orbital reconstruction at the border, and the calculations of the magnetization occurring in the superconductor due-to changes in the density of states at the border with the ferromagnet. A typical penetration depth of the magnetic moment into the superconductor is significantly greater than the coherence length of the cuprate superconductor, indicating that the induced magnetic moment mechanism of $\mathrm{Cu}$ is dominant.

It is experimentally shown that critical current in mesastructures made of layers with an interlayer that consists of a spin valve LSMO/SRO, is observed when the total layer thickness is up to $50 \mathrm{~nm}$, i.e., the thickness of the interlayer must be significantly greater than the coherence length. The maximum value of the critical current density is observed at interlayer thicknesses that are close to the coherence length of the ferromagnetic films. In fields that are less than the saturation field of the manganite ferromagnetic film, the Fourier-analysis of the magneto-field dependences allows us to select the components that correspond to the fractional values of the basic period of critical current oscillations due to the magnetic field. Such oscillations arise due to the deviation of the superconducting current CPD from the sinusoidal form. This is confirmed by microwave measurements of the Shapiro step heights as a function of irradiation power: a large value of the CPD second harmonic is detected, up to $50 \%$ of the critical current. Another factor affecting the magneto-field dependence of the critical current is the manifestation of domains. However, the mechanism behind this effect is still unclear.

The authors wish to thank V. A. Atsarkin, I. V. Borisenko, D. Winkler, A. F. Volkov, A. V. Zaitsev, B. Keimer, A. I. Kalabykhov, Yu. V. Kislinskiy, G. Logvenov, A. M. Petrizhk, A. V. Shadrin, and A. E. Scheuermann for their assistance in conducting these experiments, and the useful discussions. This study was supported by the Russian Academy of Science, RFBR Project Nos. 14-07-00258 and 14-07-93105, with equipment from the Swedish National Center for Micro and Nanotechnology (Myfab). The neutron measurements were conducted using NREX equipment, serviced by the Max Planck scientific community at the Heinz Maier-Leibnitz Zentrum in Garching, Germany.

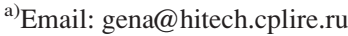

${ }^{1}$ A. I. Buzdin. Rev. Mod. Phys. 77, 935 (2005).

${ }^{2}$ G. Deutscher and P. G. de Gennes, "Proximity effects" in Superconductivity, edited by R. D. Parks (Marcel Dekker, New York, 1969), Vol. 2, p. 1005.

${ }^{3}$ P. G. de Gennes, Phys. Lett. 23, 10 (1966).

${ }^{4}$ A. I. Larkin and Yu. N. Ovchinnikov, JETP 47, 1136 (1964).

${ }^{5}$ P. Fulde and R. Ferrell, Phys. Rev. 135, A550 (1964).

${ }^{6}$ A. I. Buzdin, L. N. Bulayevskiy, and S. V. Panyukov, JETP Lett. 35, 147 (1982).

${ }^{7}$ V. V. Ryazanov, V. A. Oboznov, A. Yu. Rusanov, A. V. Veretennikov, A. A. Golubov, and J. Aarts, Phys. Rev. Lett. 86, 2427 (2001).

${ }^{8}$ F. S. Bergeret, A. F. Volkov, and K. B. Efetov, Phys. Rev. Lett. 86, 4096 (2001).

${ }^{9}$ A. Kadigrobov, R. I. Shekhter, and M. Jonson, Europhys. Lett. 54, 394 (2001).

${ }^{10}$ J. W. A. Robinson, J. D. S. Witt, and M. G. Blamire, Science 329, 59 (2010).

${ }^{11}$ M. S. Anwar, F. Czeschka, M. Hesselberth, M. Porcu, and J. Aarts, Phys. Rev. B 82, 100501 (2010).

${ }^{12}$ L. Trifunovic, Z. Popovi'c, and Z. Radovi'c, Phys. Rev. B 84, 064511 (2011).

${ }^{13}$ C. Richard, M. Houzet, and J. S. Meyer, Phys. Rev. Lett. 110, 217004 (2013).

${ }^{14}$ L. Trifunovic, Phys. Rev. Lett. 107, 047001 (2011).

${ }^{15}$ S. Yunoki, A. Moreo, E. Dagotto, S. Okamoto, and S. S. Kancharla, Phys. Rev. B 76, 064532 (2007).

${ }^{16}$ C. Visani, Z. Sefrioui, J. Tornos, C. Leon, J. Briatico, M. Bibes, A. Barthélémy, J. Santamaria, and J. E. Villegas, Nat. Phys. 8, 539 (2012).

${ }^{17}$ T. Hu, H. Xiao, C. Visani, Z. Sefrioui, J. Santamaria, and C. C. Almasan, Phys. Rev. B 80, 060506R (2009).

${ }^{18}$ Y. Kalcheim, T. Kirzhner, G. Koren, and O. Millo, Phys. Rev. B 83, 064510 (2011).

${ }^{19}$ T. Golod, A. Rydh, V. M. Krasnov, I. Marozau, M. A. UribeLaverde, D. K. Satapathy, Th. Wagner, and C. Bernhard, Phys. Rev. B 87, 134520 (2013).

${ }^{20}$ M. van Zalk, A. Brinkman, J. Aarts, and H. Hilgenkamp, Phys. Rev. B 82, 134513 (2010).

${ }^{21}$ A. M. Petrzhik, G. A. Ovsyannikov, A. V. Shadrin, K. Y. Constantinin, A. V. Zaitsev, V. V. Demidov, and Yu. V. Kislinskiy, JETP 139, 1190 (2011).

22"Magnetic heterostructures, advances and perspectives," in Spinstructures and Spintransport, Springer Tracts in Modern Physics, edited by H. Zabel and S. D. Bader (Springer/Berlin, Heidelberg, 2008), Vol. 227, p. 251.

${ }^{23}$ W. L. Lim, N. Ebrahim-Zadeh, J. C. Owens, H. G. E. Hentschel, and S. Urazhdin, Appl. Phys. Lett. 102, 162404 (2013).

${ }^{24}$ K. Halterman and O. T. Valls, Phys. Rev. B 66, 224516 (2002).

${ }^{25}$ R. Fazio and C. Lucheroni, Europhys. Lett. 45, 707 (1999).

${ }^{26}$ V. N. Krivoruchko and E. A. Koshina, Phys. Rev. B 66, 0145621 (2002).

${ }^{27}$ F. S. Bergeret, A. F. Volkov, and K. B. Efetov, Phys. Rev. B 69, 174504 (2004). 
${ }^{28}$ F. S. Bergeret, A. L. Yeyati, and A. Martin-Rodero, Phys. Rev. B 72, 064524 (2005).

${ }^{29}$ M. Yu. Kharitonov, A. F. Volkov, and K. B. Efetov, Phys. Rev. B 73, 054511 (2006).

${ }^{30}$ R. Grein, T. Löfwander, and M. Eschrig, Phys. Rev. 88, 054502 (2013).

${ }^{31}$ M. Alidoust, K. Halterman, and J. Linder, Phys. Rev. B 89, 054508 (2014).

${ }^{32}$ I. A. Garifullin, D. A. Tikhonov, N. N. Garif'yanov, M. Z. Fattakhov, K. Theis-Brцhl, K. Westerholt, and H. Zabel, Appl. Magn. Reson. 22, 439 (2002).

${ }^{33}$ M. G. Flokstra, S. J. Ray, S. J. Lister, J. Aarts, H. Luetkens, T. Prokscha, A. Suter, E. Morenzoni, and S. L. Lee, Phys. Rev. B 89, 054510 (2014).

${ }^{34}$ J. Xia, V. Shelukhin, M. Karpovski, A. Kapitulnik, and A. Palevski, Phys. Rev. Lett. 102, 087004 (2009).

${ }^{35}$ R. I. Salikhov, I. A. Garifullin, N. N. Garif'yanov, L. R. Tagirov, K. Theis-Bröhl, K. Westerholt, and H. Zabel, Phys. Rev. Lett. 102, 087003 (2009).

${ }^{36}$ J. Stahn, J. Chakhalian, Ch. Niedermayer, J. Hoppler, T. Gutberlet, J. Voigt, F. Treubel, H.-U. Habermeier, G. Cristiani, B. Keimer, and C. Bernhard, Phys. Rev. B 71, 140509(R) (2005).

${ }^{37}$ D. K. Satapathy, M. A. Uribe-Laverde, I. Marozau, V. K. Malik, S. Das, Th. Wagner, C. Marcelot, J. Stahn, S. Bruück, A. Ruühm, S. Macke, T. Tietze, E. Goering, A. Frañó, J.-H. Kim, M. Wu, E. Benckiser, B. Keimer, A. Devishvili, B. P. Toperverg, M. Merz, P. Nagel, S. Schuppler, and C. Bernhard, Phys. Rev. Lett. 108, 197201 (2012).

${ }^{38}$ J. Chakhalian, J. W. Freeland, G. Srajer, J. Strempfer, G. Khaliullin, J. C. Cezar, T. Charlton, R. Dalgliesh, C. Bernhard, G. Cristiani, H.-U. Habermeier, and B. Keimer, Nat. Phys. 2, 244 (2006).

${ }^{39}$ J. Chakhalian, J. W. Freeland, H.-U. Habermeier, G. Khaliullin, M. van Veenendaal, and B. Keimer, Science 318, 1114 (2007).

${ }^{40}$ H.-U. Habermeier, J. Phys.: Conf. Ser. 108, 012039 (2008).

${ }^{41}$ J. Santamaria, J. Garcia-Barriocanal, Z. Sefrioul, and C. Leon, Intern. J. Modern Phys. B 27, 1330013 (2013).

${ }^{42}$ J. Salafranca and S. Okamoto, Phys. Rev. Lett. 105, 256804 (2010).

${ }^{43}$ G. A. Ovsyannikov, A. E. Scheuerman, A. V. Shadrin, Yu. V. Kislinksiy, K. Y. Constantinin, and A. Kalabykhov, JETP Lett. 97, 145 (2013).

${ }^{44}$ V. V. Demidov, I. V. Borisenko, A. A. Klimov, G. A. Ovsyannikov, A. M. Petrzhik, and S. A. Nikitov, Zh. Eksp. Teor. Fiz. 139, 943 (2011).

${ }^{45}$ G. Koster, L. Klein, W. Siemons, G. Rijnders, J. Steven Dodge, C.-B. Eom, D. H. A. Blank, and M. R. Beasley, Rev. Mod. Phys. 84, 253 (2012).

${ }^{46}$ See https://www.qdusa.com/products/mpms3.html.

${ }^{47}$ Yu. N. Khaydukov, B. Nagy, J.-H. Kim, T. Keller, A. Ruühm, Yu. V. Nikitenko, K. N. Zhernenkov, J. Stahn, L. F. Kiss, A. Csik, L. Bottyán, and V. L. Aksenov, JETP Lett. 98, 107 (2013).

${ }^{48}$ H. Zabel, K. Theis Bröhl, and B. P. Toperver, Handbook of Magnetism and Advanced Magnetic Materials (John Wiley \& Sons, 2007).

${ }^{49}$ B. F. Woodfield, M. L. Wilson, and J. M. Byers, Phys. Rev. Lett. 78, 3201 (1997).

${ }^{50}$ I. Asulin, O. Yuli, G. Koren, and O. Millo, Phys. Rev. B 79, 174524 (2009).

${ }^{51}$ Yu. N. Khaydukov, G. A. Ovsyannikov, A. E. Sheyerman, K. Y. Constantinian, L. Mustafa, T. Keller, M. A. Uribe-Laverde, Yu. V. Kislinskii, A. V. Shadrin, A. Kalaboukhov, B. Keimer, and D. Winkler, Phys. Rev. B 90, 035130 (2014).
${ }^{52}$ A. E. Sheyerman, K. Y. Constantinian, G. A. Ovsyannikov, Yu. V. Kislinskii, A. V. Shadrin, A. V. Kalabukhov, and Yu. N. Khaydukov, J. Exp. Theor. Phys. 120, 1024 (2015).

${ }^{53}$ H.-U. Habermeier and G. Cristiani, Physica C 408-410, 864 (2004).

${ }^{54}$ P. Padhan, W. Prellier, and R. C. Budhani, Appl. Phys. Lett. 88, 192509 (2006).

${ }^{55}$ M. Ziese, I. Vrejoiu, E. Pippel, P. Esquinazi, D. Hesse, C. Etz, J. Henk, A. Ernst, I. V. Maznichenko, W. Hergert, and I. Mertig, Phys. Rev. Lett. 104, 167203 (2010)

${ }^{56}$ A. Y. Borisevich, A. R. Lupini, J. He, E. A. Eliseev, A. N. Morozovska, G. S. Svechnikov, P. Yu, Y.-H. Chu, R. Ramesh, S. T. Pantelides, S. V. Kalinin, and S. J. Pennycook, Phys. Rev. B 86, 140102(R) (2012).

${ }^{57}$ M. Ziese, F. Bern, A. Setzer, E. Pippel, D. Hesse, and I. Vrejoiu, Eur. Phys. J. B 86, 42 (2013).

${ }^{58}$ V. N. Menshov and V. V. Tygyshev, JETP 125, 136 (2004).

${ }^{59}$ N. M. Kreines, FNT 28, 807 (2002) [Low Temp. Phys. 28, 581 (2002)].

${ }^{60}$ N. Pugach and A. I. Buzdin, Appl. Phys. Lett. 101, 242602 (2012).

${ }^{61} \mathrm{Yu}$. N. Khaidukov and Yu. V. Nikitenko, Nucl. Instrum. Methods Phys. Res. A 629, 245 (2011).

${ }^{62}$ P. Komissinskiy, G. A. Ovsyannikov, K. Y. Constantinian, Y. V. Kislinski, I. V. Borise4nko, I. I. Soloviev, V. K. Kornev, E. Goldobin, and D. Winkler, Phys. Rev. B 78, 024501 (2008).

${ }^{63}$ L. Mieville, D. Worledge, T. H. Geballe, R. Contreras, and K. Char, Appl. Phys. Lett. 73, 1736 (1998).

${ }^{64}$ M. van Zalk, A. Brinkman, J. Aarts, and H. Hilgenkamp, Phys. Rev. B 82, 134513 (2010).

${ }^{65}$ M. A. Khasawneh, T. S. Khaire, C. Klose, W. P. Pratt, Jr., and N. O. Birge, Supercond. Sci. Technol. 24, 024005 (2011).

${ }^{66}$ A. F. Volkov and K. B. Efetov, Phys. Rev. B 81, 144522 (2010).

${ }^{67}$ J. L. Cohn, J. J. Neumeier, C. P. Popoviciu, K. J. McClellan, and Th. Leventouri, Phys. Rev. B 56, R8495 (1997).

${ }^{68}$ P. Kostic, Y. Okada, N. C. Collins, Z. Schlesinger, J. W. Reiner, L. Klein, A. Kapitulnik, T. H. Geballe, and M. R. Beasley, Phys. Rev. Lett. 81, 2498 (1998).

${ }^{69}$ A. Barone and J. Paterno, Josephson Effect. Physics and Application (Mir, Moscow, 1984).

${ }^{70}$ A. Pal, Z. H. Barber, J. W. A. Robinson, and M. G. Blamire, Nat. Commun. 5, 3340 (2014).

${ }^{71}$ F. V. Komissinskiy, G. A. Ovsyannikov, and Z. G. Ivanov, FTT 43, 769 (2000).

${ }^{72}$ Yu. V. Kislinksiy, K. I. Konstantinin, G. A. Ovsyannikov, F. V. Komissinskiy, I. V. Borisenko, and A. V. Shadrin, JETP 133, 914 (2008).

${ }^{73}$ C. Klose, T. S. Khaire, Y. Wang, W. P. Pratt, Jr., N. O. Birge, B. J. McMorran, T. P. Ginley, J. A. Borchers, B. J. Kirby, B. B. Maranville, and J. Unguris, Phys. Rev. Lett. 108, 127002 (2012).

${ }^{74}$ G. Wild, C. Probst, A. Marx, and R. Gross, Eur. Phys. J. B 78, 509 (2010).

${ }^{75}$ A. F. Volkov and K. B. Efetov, Phys. Rev. Lett. 102, 077002 (2009).

${ }^{76}$ A. I. Buzdin, A. S. Mel'nikov, and N. G. Pugach, Phys. Rev. B 83, 144515 (2011).

Translated by A. Bronskaya 